\title{
Series Solution for Steady Heat Transfer in a Heat-Generating Fin with Convection and Radiation
}

\author{
Fazle Mabood, ${ }^{1}$ Waqar A. Khan, ${ }^{2}$ and Ahmad Izani Md Ismail ${ }^{1}$ \\ ${ }^{1}$ School of Mathematical Sciences, Universiti Sains Malaysia, 11800 Penang, Malaysia \\ ${ }^{2}$ Department of Engineering Sciences, National University of Sciences and Technology, PN Engineering College, Karachi 75350, Pakistan
}

Correspondence should be addressed to Fazle Mabood; mabood1971@yahoo.com

Received 27 May 2013; Accepted 7 August 2013

Academic Editor: Farzad Khani

Copyright (C) 2013 Fazle Mabood et al. This is an open access article distributed under the Creative Commons Attribution License, which permits unrestricted use, distribution, and reproduction in any medium, provided the original work is properly cited.

\begin{abstract}
The steady heat transfer in a heat-generating fin with simultaneous surface convection and radiation is studied analytically using optimal homotopy asymptotic method (OHAM). The steady response of the fin depends on the convection-conduction parameter, radiation-conduction parameter, heat generation parameter, and dimensionless sink temperature. The heat transfer problem is modeled using two-point boundary value conditions. The results of the dimensionless temperature profile for different values of convection-conduction, radiation-conduction, heat generation, and sink temperature parameters are presented graphically and in tabular form. Comparison of the solution using OHAM with homotopy analysis method (HAM) and Runge-Kutta-Fehlberg fourthfifth-order numerical method for various values of controlling parameters is presented. The comparison shows that the OHAM results are in excellent agreement with NM.
\end{abstract}

\section{Introduction}

Fins (extended surfaces) are widely used to enhance the heat transfer rate between a hot surface and its surrounding fluid. Fin applications have included the cooling of computer processors, air conditioning units, refrigerators, air-cooled engines, and oil carrying pipelines. In the past three decades, fins have gained vast recognition for cooling electronic tools as heat sinks. The subject of extended surface heat transfer is now a fully developed technology but with continuing contributions from numerous researchers. Background information on heat transfer in extended surfaces may be found in the books $[1,2]$, where the authors have presented wideranging coverage of the various facts of this technology.

Numerous mathematical models related to heat transfer in fins of various shapes with different boundary conditions are well documented in the research literature. For instance, the mathematical analysis of convective fins was first provided by Gardener [3] based on the assumption of constant conductivity and a uniform coefficient of convective heat transfer along the fin surface. Khani et al. [4] presented some exact solutions for 1D fin problem with uniform thermal conductivity and heat transfer coefficient. Khani et al. [5] also provided a series solution for 1D fin problem with constant heat transfer coefficient and temperature dependent thermal conductivity.

A variety of approximate analytical methods have been used to study the transient response of fins. Aziz and $\mathrm{Na}$ [6] presented a coordinate perturbation expansion for the response of an infinitely long fin due to a step change in the base temperature. Chang et al. [7] used the methods of optimal linearization and variational embedding, and Campo [8] utilized variational techniques to analyze radiative-convective fins under unsteady operating conditions. Solutions for transient heat transfer were constructed for fins by Onur [9]. Aziz and Torabi [10] have presented the numerical analysis of transient heat transfer in fin with temperature dependent heat transfer coefficient.

Exact steady-state solutions of 2D models of fin having constant thermal conductivity and heat transfer coefficient and with no internal heat generation were analyzed in [11-17]. The addition of internal heat generation function based with spatial dependence is discussed [18-20].

In this paper, we used a new approximate method, namely, optimal homotopy asymptotic method [21-27] for steady-state heat transfer with internal heat generation fin, 


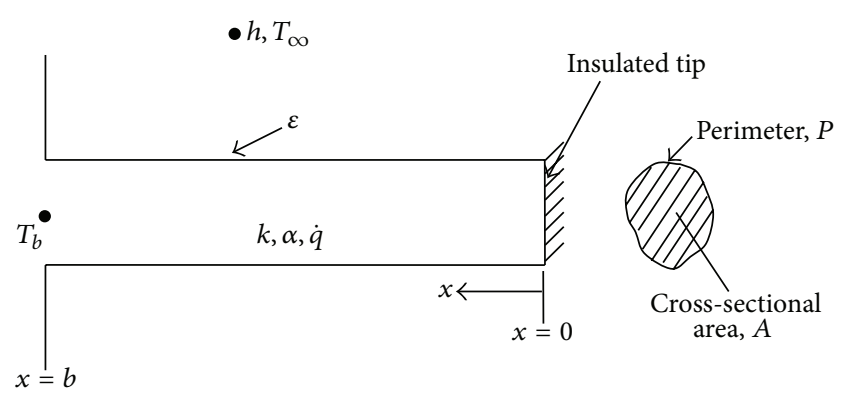

FIGURE 1: A straight fin with constant cross-sectional area.

and investigated numerically the effects of the different governing parameters on dimensionless temperature profile in a nonlinear fin-type problem. For comparison purposes the governing highly nonlinear problem is also solved using Runge-Kutta-Fehlberg fourth-fifth-order method and homotopy analysis method (HAM) developed by Liao [28].

The paper is planned as follows: in Section 2 we formulate our nonlinear problem, basic principles of OHAM are discussed in Section 3, solution of the problem via OHAM is presented in Section 4, and Section 5 is reserved for results and discussion. Conclusions are drawn in Section 6.

\section{Mathematical Formulation}

Consider a straight fin of constant cross-sectional area $A$ (rectangular, cylindrical, elliptic, etc.), perimeter of the crosssection $P$, and length $b$ as shown in Figure 1. The fin has a thermal conductivity $k$ and a thermal diffusivity $\alpha$. The surface of the fin behaves as a gray diffuse surface with an emissivity $\varepsilon$. The fin is deemed to be initially in thermal equilibrium with the surroundings at temperature $T_{s}$. Its tip is insulated. A volumetric internal heat generation rate $\dot{q}$ occurs in the fin. The fin loses heat by simultaneous convection and radiation to its surroundings at temperature $T_{s}$. The same sink temperature is used for both convection and radiation to avoid the introduction of an additional parameter in the problem.

For one-dimensional steady conduction in the fin, the energy equation may be written as

$$
\frac{\partial^{2} T}{\partial x^{2}}-\frac{h P}{k A}\left(T-T_{s}\right)-\frac{\varepsilon \sigma P\left(T^{4}-T_{s}^{4}\right)}{k A}+\frac{\dot{q}}{k}=0 .
$$

The initial and boundary conditions are

$$
\begin{aligned}
& T(x)=T_{s}, \\
& T(b)=T_{b}, \\
& \frac{d T}{d x}(0)=0,
\end{aligned}
$$

where $x$ is measured from the tip of the fin with the introduction of the following definitions:

$$
\begin{gathered}
\theta=\frac{T}{T_{b}}, \quad \theta_{s}=\frac{T_{s}}{T_{b}}, \quad X=\frac{x}{b}, \quad h_{b}=C\left(T_{b}-T_{s}\right), \\
N_{c}=\frac{h_{b} P b^{2}}{k A}, \quad N_{r}=\frac{\varepsilon \sigma P T_{s}^{3} b^{2}}{k A}, \\
Q_{\text {gen }}=\frac{\dot{q} b^{2}}{k T_{b}} .
\end{gathered}
$$

Equations (2) and (3) can be written in dimensionless form as follows:

$$
\begin{gathered}
\frac{d^{2} \theta}{d X^{2}}-\frac{N_{c}}{\left(1-\theta_{s}\right)}\left(\theta-\theta_{s}\right)^{2}-N_{r}\left(\theta^{4}-\theta_{s}^{4}\right)+Q_{\text {gen }}=0, \\
\theta(b)=1, \\
\frac{d \theta}{d X}(0)=0 .
\end{gathered}
$$

The instantaneous base heat flow is given by:

$$
q_{b}=k A \frac{d T}{d x}(b),
$$

which may be expressed in dimensionless form as follows.

$$
Q_{b}=\frac{q_{b} b}{k A T_{b}}=\frac{d \theta}{d X}(1) .
$$

The instantaneous convective heat loss from the fin is given by

$$
q_{c}=P \int_{0}^{b} h\left(T-T_{s}\right) d x,
$$

or in dimensionless form as

$$
Q_{c}=\frac{q_{c} b}{k A T_{b}}=\frac{N_{c}}{\left(1-\theta_{s}\right)} \int_{0}^{1}\left(\theta-\theta_{s}\right)^{2} d X .
$$

Similarly, the instantaneous radiative heat loss from the fin can be obtained as

$$
q_{r}=\varepsilon \sigma P \int_{0}^{b}\left(T^{4}-T_{s}^{4}\right) d x,
$$

or in dimensionless form as

$$
Q_{r}=\frac{q_{r} b}{k A T_{b}}=N_{r} \int_{0}^{1}\left(\theta^{4}-\theta_{s}^{4}\right) d X
$$

The instantaneous total surface heat loss in dimensionless form is the sum of convective and radiative losses given by (11) and (13); that is,

$$
Q_{\text {loss }}=Q_{c}+Q_{r} .
$$


The instantaneous rate of energy storage in the fin can be calculated from the energy balance as follows:

$$
q_{\text {stored }}=q_{b}+q_{\text {gen }}-q_{\text {loss }}
$$

or in dimensionless form as

$$
Q_{\text {stored }}=Q_{b}+Q_{\text {gen }}-Q_{\text {loss }},
$$

where

$$
Q_{\text {gen }}=\frac{\dot{q} b^{2}}{k T_{b}}
$$

\section{Basic Principles of OHAM}

We review the basic principles of OHAM as expounded by Marinca et al. [21-24] as well as other researchers including $[25,26]$.

(i) Let us consider the following differential equation:

$$
\mathfrak{I}[\mathrm{v}(\omega)]+a(\omega)=0, \quad x \in \Omega,
$$

where $\Omega$ is problem domain, $\mathfrak{I}(\mathrm{v})=L(\mathrm{v})+N(\mathrm{v})$, where $L$ and $N$ are linear and nonlinear operators, $\mathrm{v}(x)$ is an unknown function, and $a(\omega)$ is a known function.

(ii) Construct an optimal homotopy equation as

$(1-p)[L(\phi(\omega ; p))+a(\omega)]-H(p)[\mathfrak{I}(\phi(\omega ; p))+a(\omega)]=0$,

where $0 \leq p \leq 1$ is an embedding parameter, and $H(p)=\sum_{k=1}^{m} p^{k} C_{k}$ is auxiliary function on which the convergence of the solution greatly depends. The auxiliary function $H(p)$ also adjusts the convergence domain and controls the convergence region.

(iii) Expand $\phi\left(\omega ; p, C_{j}\right)$ in Taylor's series about $p$. One has an approximate solution:

$\phi\left(\omega ; p, C_{j}\right)=\mathrm{v}_{0}(\omega)+\sum_{k=1}^{\infty} \mathrm{v}_{k}\left(\omega, C_{j}\right) p^{k}, \quad j=1,2,3, \ldots$

Many researchers have observed that the convergence of the series equation (19) depends upon $C_{j},(j=$ $1,2, \ldots, m)$. If it is convergent, then we obtain

$$
\widetilde{\mathrm{v}}=\mathrm{v}_{0}(\omega)+\sum_{k=1}^{m} \mathrm{v}_{k}\left(\omega ; C_{j}\right)
$$

(iv) Substituting (20) in (17), we have the following residual:

$R\left(\omega ; C_{j}\right)=L\left(\widetilde{\mathrm{v}}\left(\omega ; C_{j}\right)\right)+a(\omega)+N\left(\widetilde{\mathrm{v}}\left(\omega ; C_{j}\right)\right)$.

If $R\left(\omega ; C_{j}\right)=0$, then $\widetilde{v}$ will be the exact solution. For nonlinear problems, generally this will not be the case. For determining $C_{j}(j=1,2, \ldots, m)$, Galerkin's Method, Ritz Method, or the method of least squares can be used.

(v) Finally, substitute these constants in (21), and one can get the approximate solution.

\section{OHAM Solution for Heat-Generating Fin}

According to the OHAM, (1) can be written as

$$
\begin{aligned}
& (1-p)\left(\theta^{\prime \prime}\right)-H(p) \\
& \quad \times\left(\theta^{\prime \prime}-\frac{N_{c}}{\left(1-\theta_{s}\right)}\left(\theta-\theta_{s}\right)^{2}-N_{r}\left(\theta^{4}-\theta_{s}^{4}\right)+Q_{g}\right)=0,
\end{aligned}
$$

where prime denotes differentiation with respect to $X$.

We consider $\theta$ and $H(p)$ as follows:

$$
\begin{aligned}
& \theta=\theta_{0}+p \theta_{1}+p^{2} \theta_{2}, \\
& H(p)=p C_{1}+p^{2} C_{2} .
\end{aligned}
$$

Using (23) in (22) and after some simplifying and rearranging the terms based on the powers of $p$, we obtain the zeroth-, first-, and second-order problems as follows.

The zeroth-order problem is

$$
\frac{d^{2} \theta_{0}(X)}{d X^{2}}=0
$$

with boundary conditions

$$
\theta_{0}(b)=1, \quad \frac{d \theta_{0}(0)}{d X}=0 .
$$

Its solution is

$$
\theta_{0}(X)=1 \text {. }
$$

The first-order problem is

$$
\begin{gathered}
\frac{d^{2} \theta_{1}\left(X, C_{1}\right)}{d X^{2}}-C_{1} Q_{g}+\frac{C_{1} N_{c} \theta_{s}^{2}}{1-\theta_{s}}-C_{1} N_{r} \theta_{s}^{4}-\frac{2 C_{1} N_{c} \theta_{s} \theta_{0}}{1-\theta_{s}} \\
+\frac{C_{1} N_{c} \theta_{0}^{2}}{1-\theta_{s}}+C_{1} N_{r} \theta_{0}^{4}-\left(1+C_{1}\right) \frac{d^{2} \theta_{0}(X)}{d X^{2}}=0
\end{gathered}
$$

with boundary conditions

$$
\theta_{1}(b)=0, \quad \frac{d \theta_{1}(0)}{d X}=0
$$

having solution

$$
\begin{aligned}
\theta_{1}\left(X, C_{1}\right)=\frac{1}{2}( & b^{2} C_{1} N_{c}-X^{2} C_{1} N_{c}+b^{2} C_{1} N_{r}-X^{2} C_{1} N_{r} \\
& -b^{2} C_{1} Q_{g}+X^{2} C_{1} Q_{g}-b^{2} C_{1} N_{c} \theta_{s} \\
& \left.+X^{2} C_{1} N_{c} \theta_{s}-b^{2} C_{1} N_{r} \theta_{s}^{4}+X^{2} C_{1} N_{r} \theta_{s}^{4}\right) .
\end{aligned}
$$


TABLE 1: Comparison of percentage error between OHAM, HAM, and NM for temperature at $N_{c}=0.1, N_{r}=0.1$, and $\theta_{s}=0.01$.

\begin{tabular}{|c|c|c|c|c|c|c|c|c|c|c|}
\hline \multirow{2}{*}{$X$} & \multicolumn{5}{|c|}{$Q_{g}=0$} & \multicolumn{5}{|c|}{$Q_{g}=1$} \\
\hline & OHAM & HAM & NM & OHAM error & HAM error & OHAM & HAM & NM & OHAM error & HAM error \\
\hline 0 & 0.9078 & 0.9075 & 0.9078 & 0 & 0.033 & 1.8085 & 1.8081 & 1.8085 & 0 & 0.022 \\
\hline 0.1 & 0.9088 & 0.9081 & 0.9087 & 0.011 & 0.066 & 1.8005 & 1.8011 & 1.8007 & 0.011 & 0.022 \\
\hline 0.2 & 0.9115 & 0.9112 & 0.9115 & 0 & 0.032 & 1.7763 & 1.7759 & 1.7770 & 0.039 & 0.061 \\
\hline 0.3 & 0.9161 & 0.9158 & 0.9161 & 0 & 0.032 & 1.7358 & 1.7352 & 1.7375 & 0.098 & 0.132 \\
\hline 0.4 & 0.9226 & 0.9220 & 0.9224 & 0.021 & 0.043 & 1.6792 & 1.6788 & 1.6821 & 0.172 & 0.398 \\
\hline 0.5 & 0.9308 & 0.9302 & 0.9306 & 0.021 & 0.042 & 1.6065 & 1.6062 & 1.6105 & 0.248 & 0.266 \\
\hline 0.6 & 0.9410 & 0.9413 & 0.9407 & 0.032 & 0.063 & 1.5175 & 1.5181 & 1.5224 & 0.321 & 0.374 \\
\hline 0.7 & 0.9529 & 0.9522 & 0.9526 & 0.031 & 0.041 & 1.4124 & 1.4111 & 1.4176 & 0.366 & 0.458 \\
\hline 0.8 & 0.9668 & 0.9671 & 0.9665 & 0.031 & 0.062 & 1.2911 & 1.2909 & 1.2958 & 0.362 & 0.378 \\
\hline 0.9 & 0.9825 & 0.9818 & 0.9822 & 0.031 & 0.04 & 1.1536 & 1.1521 & 1.1567 & 0.268 & 0.397 \\
\hline 1.0 & 1 & 1 & 1 & 0 & 0 & 1 & 1 & 1 & 0 & 0 \\
\hline
\end{tabular}

TABLE 2: Comparison of percentage error between OHAM, HAM, and NM for temperature at $N_{r}=0.5, N_{c}=0.5$, and $Q_{g}=2$.

\begin{tabular}{|c|c|c|c|c|c|c|c|c|c|c|}
\hline \multirow{2}{*}{$X$} & \multicolumn{5}{|c|}{$\theta_{s}=0.01$} & \multicolumn{5}{|c|}{$\theta_{s}=0.4$} \\
\hline & OHAM & HAM & NM & OHAM error & HAM error & OHAM & HAM & NM & OHAM error & HAM error \\
\hline 0 & 1.3389 & 1.3373 & 1.3383 & 0.044 & 0.074 & 1.3886 & 1.3878 & 1.3886 & 0 & 0.057 \\
\hline 0.1 & 1.3357 & 1.3350 & 1.3352 & 0.277 & 0.014 & 1.3847 & 1.3844 & 1.3852 & 0.036 & 0.057 \\
\hline 0.2 & 1.3256 & 1.3248 & 1.3260 & 0.031 & 0.090 & 1.3731 & 1.3727 & 1.3748 & 0.123 & 0.152 \\
\hline 0.3 & 1.3086 & 1.3068 & 1.3106 & 0.152 & 0.289 & 1.3537 & 1.3540 & 1.3574 & 0.272 & 0.250 \\
\hline 0.4 & 1.2849 & 1.2821 & 1.2887 & 0.295 & 0.512 & 1.3265 & 1.3247 & 1.3326 & 0.458 & 0.592 \\
\hline 0.5 & 1.2544 & 1.2543 & 1.2600 & 0.444 & 0.452 & 1.2915 & 1.2911 & 1.3000 & 0.654 & 0.684 \\
\hline 0.6 & 1.2171 & 1.2156 & 1.2243 & 0.588 & 0.710 & 1.2487 & 1.2472 & 1.2591 & 0.826 & 0.945 \\
\hline 0.7 & 1.1729 & 1.1717 & 1.1809 & 0.677 & 0.779 & 1.2012 & 1.2010 & 1.2095 & 0.686 & 0.702 \\
\hline 0.8 & 1.1221 & 1.1212 & 1.1295 & 0.655 & 0.734 & 1.1399 & 1.1386 & 1.1402 & 0.796 & 0.140 \\
\hline 0.9 & 1.0644 & 1.0637 & 1.0694 & 0.467 & 0.533 & 1.0738 & 1.0725 & 1.0807 & 0.638 & 0.758 \\
\hline 1.0 & 1 & 1 & 1 & 0 & 0 & 1 & 1 & 1 & 0 & 0 \\
\hline
\end{tabular}

The second-order problem is

$$
\begin{aligned}
& \frac{d^{2} \theta_{2}\left(X, C_{1}, C_{2}\right)}{d X^{2}}-C_{2} Q_{g}+\frac{C_{2} N_{c} \theta_{s}^{2}}{1-\theta_{s}}-C_{2} N_{r} \theta_{s}^{4}-\frac{2 C_{2} N_{c} \theta_{s} \theta_{0}}{1-\theta_{s}} \\
& +\frac{C_{2} N_{c} \theta_{0}^{2}}{1-\theta_{s}}+C_{2} N_{r} \theta_{0}^{4}-\frac{2 C_{1} N_{c} \theta_{s} \theta_{1}}{1-\theta_{s}}+\frac{2 C_{1} N_{c} \theta_{0} \theta_{1}}{1-\theta_{s}} \\
& +4 C_{1} N_{r} \theta_{0}^{3} \theta_{1}-C_{2} \frac{d^{2} \theta_{0}(X)}{d X^{2}}-\left(1+C_{1}\right) \frac{d^{2} \theta_{1}\left(X, C_{1}\right)}{d X^{2}}=0
\end{aligned}
$$

with boundary conditions

$$
\theta_{2}(b)=0, \quad \frac{d \theta_{2}(0)}{d X}=0 .
$$

It is given by

$$
\begin{aligned}
& \theta_{2}\left(X, C_{1}, C_{2}\right) \\
& =\frac{1}{12}\left(6 b^{2} C_{1} N_{c}-6 X^{2} C_{1} N_{c}+6 b^{2} C_{1}^{2} N_{c}-6 X^{2} C_{1}^{2} N_{c}\right. \\
& \quad+6 b^{2} C_{2} N_{c}-6 X^{2} C_{1} N_{r}-6 X^{2} C_{2} N_{c}+5 b^{4} C_{1}^{2} N_{c}^{2}
\end{aligned}
$$

$-6 b^{2} X^{2} C_{1}^{2} N_{c}^{2}+X^{4} C_{1}^{2} N_{c}^{2}+6 b^{2} C_{1} N_{r}+15 b^{4} C_{1}^{2} N_{c} N_{r}$

$+6 b^{2} C_{1}^{2} N_{r}-6 X^{2} C_{1}^{2} N_{r}+6 b^{2} C_{2} N_{r}-6 X^{2} C_{2} N_{r}$

$+10 b^{4} C_{1}^{2} N_{r}^{2}-12 b^{2} X^{2} C_{1}^{2} N_{r}^{2}-18 b^{2} X^{2} C_{1}^{2} N_{c} N_{r}$

$+3 X^{4} C_{1}^{2} N_{c} N_{r}-6 b^{2} C_{1}^{2} Q_{g}+6 X^{2} C_{1}^{2} Q_{g}-6 b^{2} C_{2} Q_{g}$

$+2 X^{4} C_{1}^{2} N_{r}^{2}-6 b^{2} C_{1} Q_{g}+6 X^{2} C_{1} Q_{g}+6 X^{2} C_{2} Q_{g}$

$-5 b^{4} C_{1}^{2} N_{c} Q_{g}+6 b^{2} X^{2} C_{1}^{2} N_{c} Q_{g}-X^{4} C_{1}^{2} N_{c} Q_{g}$

$-10 b^{4} C_{1}^{2} N_{r} Q_{g}+12 b^{2} X^{2} C_{1}^{2} N_{r} Q_{g}-2 X^{4} C_{1}^{2} N_{r} Q_{g}$

$-6 b^{2} C_{1} N_{c} \theta_{s}+6 X^{2} C_{1} N_{c} \theta_{s}-6 b^{2} C_{1}^{2} N_{c} \theta_{s}+6 X^{2} C_{1}^{2} N_{c} \theta_{s}$

$-6 b^{2} C_{2} N_{c} \theta_{s}+6 X^{2} C_{2} N_{c} \theta_{s}-X^{4} C_{1}^{2} N_{c}^{2} \theta_{s}-5 b^{4} C_{1}^{2} N_{c}^{2} \theta_{s}$

$+6 b^{2} X^{2} C_{1}^{2} N_{c}^{2} \theta_{s}-10 b^{4} C_{1}^{2} N_{c} N_{r} \theta_{s}+12 b^{2} X^{2} C_{1}^{2} N_{c} N_{r} \theta_{s}$

$-6 b^{2} C_{2} N_{r} \theta_{s}^{4}-2 X^{4} C_{1}^{2} N_{c} N_{r} \theta_{s}-6 b^{2} C_{1} N_{r} \theta_{s}^{4}$

$+6 X^{2} C_{1} N_{r} \theta_{s}^{4}-6 b^{2} C_{1}^{2} N_{r} \theta_{s}^{4}$

$+6 X^{2} C_{1}^{2} N_{r} \theta_{s}^{4}+6 X^{2} C_{2} N_{r} \theta_{s}^{4}-5 b^{4} C_{1}^{2} N_{c} N_{r} \theta_{s}^{4}$ 
TABLE 3: Comparison of OHAM and NM for temperature at $N_{r}=0.1, \theta_{s}=0.1$, and $Q_{g}=0$.

\begin{tabular}{|c|c|c|c|c|c|c|c|c|c|}
\hline \multirow{2}{*}{$X$} & \multicolumn{3}{|c|}{$N_{c}=0.1$} & \multicolumn{3}{|c|}{$N_{c}=0.3$} & \multicolumn{3}{|c|}{$N_{c}=1$} \\
\hline & OHAM & NM & $\%$ error & OHAM & NM & $\%$ error & OHAM & NM & $\%$ error \\
\hline 0 & 0.8172 & 0.8187 & 0.183 & 0.7623 & 0.7668 & 0.586 & 0.6409 & 0.6465 & 0.866 \\
\hline 0.1 & 0.8190 & 0.8190 & 0 & 0.7647 & 0.7691 & 0.572 & 0.6445 & 0.6496 & 0.785 \\
\hline 0.2 & 0.8245 & 0.8259 & 0.169 & 0.7718 & 0.7758 & 0.515 & 0.6552 & 0.6592 & 0.607 \\
\hline 0.3 & 0.8336 & 0.8348 & 0.143 & 0.7883 & 0.7871 & 0.152 & 0.6732 & 0.6752 & 0.296 \\
\hline 0.4 & 0.8464 & 0.8474 & 0.118 & 0.8004 & 0.8029 & 0.311 & 0.6983 & 0.6979 & 0.057 \\
\hline 0.5 & 0.8629 & 0.8636 & 0.081 & 0.8217 & 0.8234 & 0.206 & 0.7307 & 0.7375 & 0.922 \\
\hline 0.6 & 0.8830 & 0.8834 & 0.045 & 0.8579 & 0.8586 & 0.081 & 0.7696 & 0.7646 & 0.654 \\
\hline 0.7 & 0.9067 & 0.9069 & 0.022 & 0.8788 & 0.8788 & 0 & 0.8018 & 0.8096 & 0.963 \\
\hline 0.8 & 0.9341 & 0.9341 & 0 & 0.9145 & 0.9138 & 0.076 & 0.8693 & 0.8632 & 0.707 \\
\hline 0.9 & 0.9652 & 0.9651 & 0.010 & 0.9548 & 0.9541 & 0.073 & 0.9291 & 0.9263 & 0.302 \\
\hline 1.0 & 1 & 1 & 0 & 1 & 1 & 0 & 1 & 1 & 0 \\
\hline
\end{tabular}

TABLE 4: Comparison of OHAM and NM for temperature at $N_{c}=0.5, \theta_{s}=0.3$, and $Q_{g}=1$.

\begin{tabular}{lccccccccc}
\hline$X$ & & $N_{r}=0.1$ & & & $N_{r}=0.3$ & & \multicolumn{2}{c}{$N_{r}=0.5$} \\
& OHAM & NM & \% error & OHAM & NM & \% error & OHAM & NM error \\
\hline 0 & 1.1866 & 1.1869 & 0.025 & 1.1166 & 1.1204 & 0.339 & 1.0521 & 1.0522 \\
0.1 & 1.1848 & 1.1852 & 0.033 & 1.1154 & 1.1193 & 0.348 & 1.0516 & 1.0518 & 0.009 \\
0.2 & 1.1792 & 1.1801 & 0.076 & 1.1119 & 1.1160 & 0.367 & 1.0501 & 1.0503 & 0.019 \\
0.3 & 1.1698 & 1.1715 & 0.145 & 1.1061 & 1.1104 & 0.387 & 1.0477 & 1.0479 & 0.019 \\
0.4 & 1.1568 & 1.1590 & 0.189 & 1.0979 & 1.1026 & 0.426 & 1.0442 & 1.0445 & 0.028 \\
0.5 & 1.1400 & 1.1435 & 0.306 & 1.0874 & 1.0923 & 0.448 & 1.0398 & 1.0400 & 0.019 \\
0.6 & 1.1194 & 1.1237 & 0.382 & 1.0746 & 1.0795 & 0.453 & 1.0342 & 1.0345 \\
0.7 & 1.0952 & 1.0997 & 0.409 & 1.0594 & 1.0641 & 0.441 & 1.0275 & 1.0278 & 0.028 \\
0.8 & 1.0672 & 1.0713 & 0.382 & 1.0419 & 1.0458 & 0.373 & 1.0196 & 1.0198 & 0.019 \\
0.9 & 1.0354 & 1.0382 & 0.269 & 1.0221 & 1.0245 & 0.234 & 1.0104 & 1.0106 \\
1.0 & 1 & 1 & 0 & 1 & 1 & 0 & 0.019 & 1 \\
\hline
\end{tabular}

$$
\begin{aligned}
& +6 b^{2} X^{2} C_{1}^{2} N_{c} N_{r} \theta_{s}^{4}-X^{4} C_{1}^{2} N_{c} N_{r} \theta_{s}^{4}-10 b^{4} C_{1}^{2} N_{r}^{2} \theta_{s}^{4} \\
& \left.+12 b^{2} X^{2} C_{1}^{2} N_{r}^{2} \theta_{s}^{4}-2 X^{4} C_{1}^{2} N_{r}^{2} \theta_{s}^{4}\right)
\end{aligned}
$$

The second-order approximate solution by OHAM for $p=1$ is

$$
\tilde{\theta}\left(X, C_{1}, C_{2}\right)=\theta_{0}(X)+\theta_{1}\left(X, C_{1}\right)+\theta_{2}\left(X, C_{1}, C_{2}\right)
$$

We use the method of least squares to obtain $C_{1}, C_{2}$ the unknown convergent constant in $\widetilde{\theta}$.

In particular case, $C_{1}=-0.00002137$ and $C_{2}=-0.79986$, for $N_{r}=0.3, N_{c}=0.3, Q_{g}=0.4, \theta_{s}=0.2$, and $b=1$.

By considering the values of $C_{1}, C_{2}$ in (33) and after simplifying, the second-order approximate analytical OHAM solution can be obtained

$$
\begin{aligned}
\theta= & +\frac{1}{2}\left(-0.000002982+2.982 \times 10^{-6} X^{2}\right) \\
& +\frac{1}{12}\left(-0.6696+0.669596 X^{2}+5.73 \times 10^{-11} X^{4}\right) .
\end{aligned}
$$

\section{Results and Discussion}

Equation (4) shows that fin temperature is based on four parameters: $N_{r}, N_{c}, \theta_{s}$, and $Q_{g}$ which govern this highly nonlinear second-order differential equation. The effect of each parameter on fin temperature is tabulated and graphically presented for different values of the controlling parameters.

In order to validate the accuracy of our approximate solution via OHAM, we have presented a comparative study of OHAM solution with homotopy analysis method (HAM) and numerical solution (Runge-Kutta-Fehlberg fourth-fifthorder method). Table 1 has been prepared to exhibit the comparison of dimensionless temperature $\theta$ obtained by OHAM, homotopy analysis method (HAM), and the numerical method (NM) for several values of heat-generating parameter $Q_{g}$, when other parameters are fixed. It is observed that, with increasing values of internal heat-generating parameter $Q_{g}$, the temperature profile gradually increases. Clearly the OHAM solutions are very close to the numerical solution as compared to HAM. This can be seen from the percentage error in the dimensionless temperature obtained by OHAM, HAM, and NM. The increase in dimensionless temperature $\theta$ is also evident in Table 2, in which we have used different values of sink temperature parameter $\theta_{s}$, and other parameters 


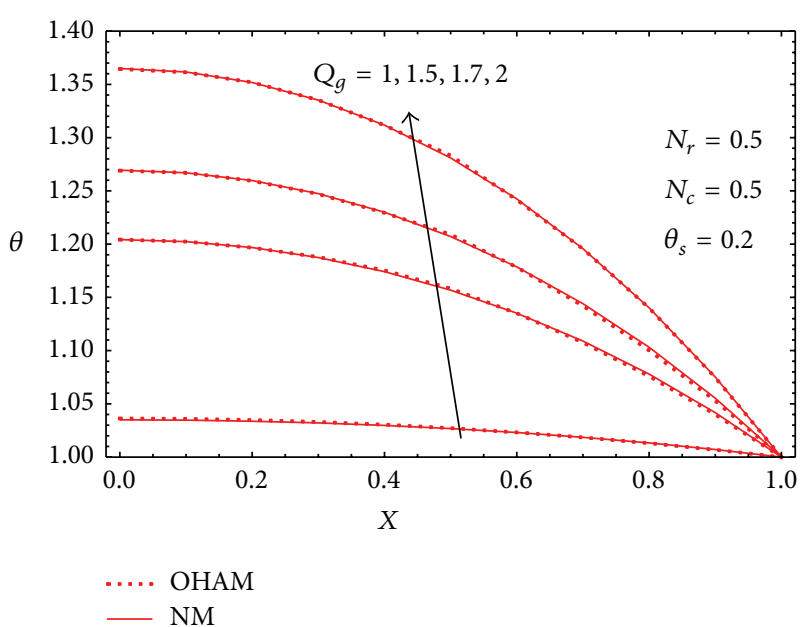

FIgURE 2: Effect of internal heat generation on fin dimensionless temperature for fixed values of $N_{r}, N_{c}$, and $\theta_{s}$.

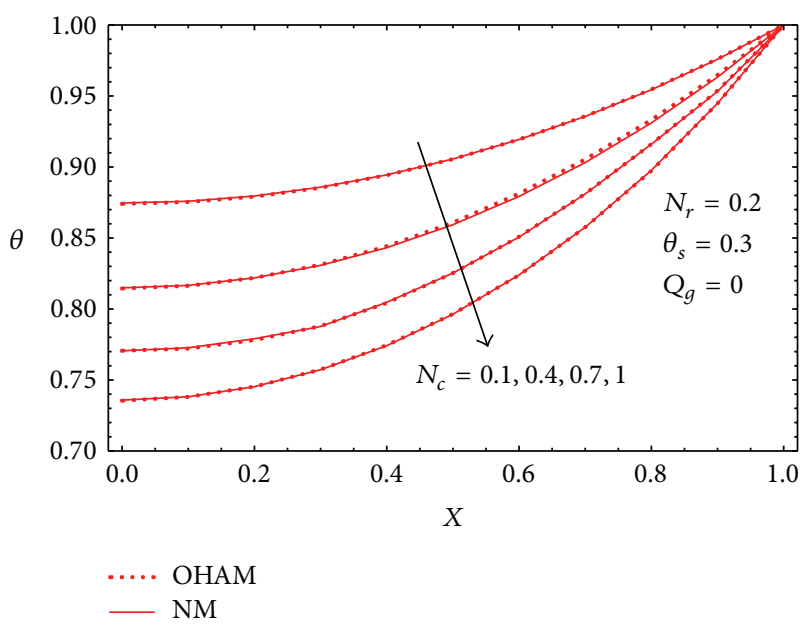

Figure 3: Effect of convection parameter on fin dimensionless temperature for fixed values of $N_{r}, \theta_{s}$, and $Q_{g}$.

values are predetermined. From Tables 1 and 2, it is observed that our OHAM solutions are more accurate than HAM; this confirms that OHAM is more consistent with approximate analytical method than with HAM. The major factor in HAM is its computational time for finding the $\hbar$ (h curve), while in OHAM the ensuring convergence of the solution depends on parameters $C_{1}, C_{2}, \ldots$, which are optimally determined, resultantly HAM in more time consuming than OHAM.

In Table 3, we show the comparison of dimensionless temperature $\theta$ obtained by OHAM and the numerical method (NM) for several values of convection parameter $N_{c}$, while other parameters are kept unchanged. It is observed that, with the increase of $N_{c}$, the temperature profile shows decrease, and the same phenomena of decrease in dimensionless temperature $\theta$ can be observed in Table 4 for different values of radiation parameter $N_{r}$, when the other parameters values are fixed.

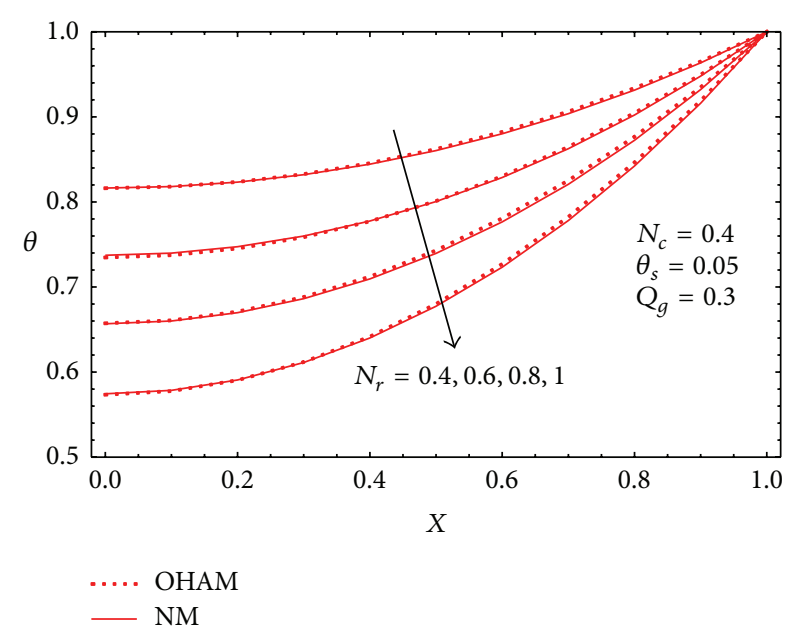

FIGURE 4: Effect of radiation parameter on fin dimensionless temperature for fixed values of $N_{c}, \theta_{s}$, and $Q_{g}$.

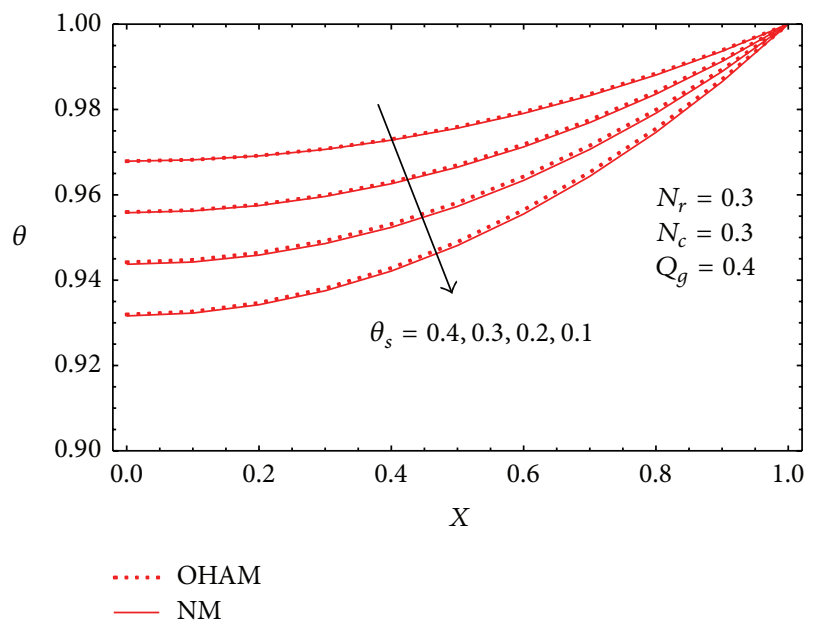

FIGURE 5: Effect of sink temperature parameter on fin dimensionless temperature for fixed values of $N_{c}, \theta_{s}$, and $Q_{g}$.

In Figures 2, 3, 4, and 5 we depict the dimensionless temperature profile $\theta$ and its variation for different values of parameters. It is important to note that the dimensionless temperature increases with each controlling parameter.

\section{Conclusion}

We have successfully applied the optimal homotopy asymptotic method for the approximate solution of steady state of heat-generating fin with simultaneous surfaces convection and radiation. The effects of radiation parameter $N_{r}$, convection parameter $N_{c}$, internal heat-generating parameter $Q_{g}$, and the sink temperature parameter $\theta_{s}$ on temperature profile in the fin are investigated analytically. It is observed that dimensionless fin temperature profile is dependent on the four parameters $N_{r}, N_{c}, Q_{g}$, and $\theta_{s}$. Comparison for the dimensionless temperature has been made between the 
solutions obtained using OHAM with HAM and RungeKutta-Fehlberg fourth-fifth-order method. It is found that the OHAM solution is very close to the numerical solution than HAM, which reveals the reliability and efficiency of OHAM. Approximate analytical solution to highly nonlinear problem was achieved without any assumption of linearization, and we can extend this approach to a variety of nonlinear heat transfer problems.

\section{References}

[1] B. Sunden and P. J. Heggs, Recent Advances in Analysis of Heat Transfer for Fin Type Surfaces, WIT Press, Boston, Mass, USA, 2000.

[2] A. D. Kraus, A. Aziz, and J. Welty, Extended Surface Heat Transfer, John Wiley \& Sons, New York, NY, USA, 2001.

[3] K. A. Gardener, "Efficiency of extended surface," Journal of Heat Transfer, vol. 67, pp. 621-631, 1945.

[4] F. Khani, M. A. Raji, and H. H. Nejad, "Analytical solutions and efficiency of the nonlinear fin problem with temperaturedependent thermal conductivity and heat transfer coefficient," Communications in Nonlinear Science and Numerical Simulation, vol. 14, no. 8, pp. 3327-3338, 2009.

[5] F. Khani, M. A. Raji, and S. Hamedi-Nezhad, "A series solution of the fin problem with a temperature-dependent thermal conductivity," Communications in Nonlinear Science and Numerical Simulation, vol. 14, no. 7, pp. 3007-3017, 2009.

[6] A. Aziz and T. Y. Na, "Transient response of fins by coordinate perturbation expansion," International Journal of Heat and Mass Transfer, vol. 23, no. 12, pp. 1695-1698, 1980.

[7] Y. M. Chang, C. K. Chen, and J. W. Cleaver, “Transient response of fins by optimal linearization and variational embedding methods," ASME Journal of Heat Transfer, vol. V 104, no. 4, pp. 813-815, 1982.

[8] A. Campo, "Variational techniques applied to radiativeconvective fins with steady and unsteady conditions," Wärmeund Stoffübertragung, vol. 9, no. 2, pp. 139-144, 1976.

[9] N. Onur, "A simplified approach to the transient conduction in a two-dimensional fin," International Communications in Heat and Mass Transfer, vol. 23, no. 2, pp. 225-238, 1996.

[10] A. Aziz and M. Torabi, "Convective-radiative fins with simultaneous variation of thermal conductivity, heat transfer coefficient, and surface emissivity with temperature," Heat TransferAsian Research, vol. 41, no. 2, pp. 99-113, 2012.

[11] L.-T. Chen, "Two-dimensional fin efficiency with combined heat and mass transfer between water-wetted fin surface and moving moist airstream," International Journal of Heat and Fluid Flow, vol. 12, no. 1, pp. 71-76, 1991.

[12] A. Aziz and H. Nguyen, "Two-dimensional performance of convecting-radiating fins of different profile shapes," Wärme und Stoffübertragung, vol. 28, no. 8, pp. 481-487, 1993.

[13] H. S. Kang and D. C. Look Jr., "Two dimensional trapezoidal fins analysis," Computational Mechanics, vol. 19, no. 3, pp. 247-250, 1997.

[14] A. Aziz and O. D. Makinde, "Heat transfer and entropy generation in a two-dimensional orthotropic convection pin fin," International Journal of Exergy, vol. 7, no. 5, pp. 579-592, 2010.

[15] S. M. Zubair, A. F. M. Arif, and M. H. Sharqawy, "Thermal analysis and optimization of orthotropic pin fins: a closed-form analytical solution," Journal of Heat Transfer, vol. 132, no. 3, pp. $1-8,2010$.

[16] Y. Xia and A. M. Jacobi, "An exact solution to steady heat conduction in a two-dimensional slab on a one-dimensional fin: application to frosted heat exchangers," International Journal of Heat and Mass Transfer, vol. 47, no. 14-16, pp. 3317-3326, 2004.

[17] M. Y. Malik and A. Rafiq, "Two-dimensional fin with convective base condition," Nonlinear Analysis. Real World Applications, vol. 11, no. 1, pp. 147-154, 2010.

[18] A. Aziz, "Effects of internal heat generation, anisotropy, and base temperature nonuniformity on heat transfer from a twodimensional rectangular fin," Heat Transfer Engineering, vol. 14, no. 2, pp. 63-70, 1993.

[19] S. Jahangeer, M. K. Ramis, and G. Jilani, "Conjugate heat transfer analysis of a heat generating vertical plate," International Journal of Heat and Mass Transfer, vol. 50, no. 1-2, pp. 85-93, 2007.

[20] M. K. Ramis, G. Jilani, and S. Jahangeer, "Conjugate conduction-forced convection heat transfer analysis of a rectangular nuclear fuel element with non-uniform volumetric energy generation," International Journal of Heat and Mass Transfer, vol. 51, no. 3-4, pp. 517-525, 2008.

[21] V. Marinca and N. Herişanu, "Application of optimal homotopy asymptotic Method for solving nonlinear equations arising in heat transfer," International Communications in Heat and Mass Transfer, vol. 35, no. 6, pp. 710-715, 2008.

[22] V. Marinca, N. Herişanu, and I. Nemeş, "Optimal homotopy asymptotic method with application to thin film flow," Central European Journal of Physics, vol. 6, no. 3, pp. 648-653, 2008.

[23] V. Marinca and N. Herişanu, "Determination of periodic solutions for the motion of a particle on a rotating parabola by means of the optimal homotopy asymptotic method," Journal of Sound and Vibration, vol. 329, no. 9, pp. 1450-1459, 2010.

[24] V. Marinca and N. Herişanu, "Accurate analytical solutions to oscillators with discontinuities and fractional-power restoring force by means of the optimal homotopy asymptotic method," Computers \& Mathematics with Applications, vol. 60, no. 6, pp. 1607-1615, 2010.

[25] S. Islam, R. A. Shah, I. Ali, and N. M. Allah, "Optimal homotopy asymptotic solutions of couette and poiseuille flows of a third grade fluid with heat transfer analysis," International Journal of Nonlinear Sciences and Numerical Simulation, vol. 11, no. 6, pp. 389-400, 2010.

[26] F. Mabood, W. A. Khan, and A. I. M. Ismail, "Optimal homotopy asymptotic method for heat transfer in hollow sphere with robin boundary conditions," Heat Transfer-Asian Research, 7 pages, 2013.

[27] F. Mabood, W. A. Khan, and A. I. M. Ismail, "Solution of Solution of nonlinear boundary layer equation for flat plate via optimal homotopy asymptotic method," Heat Transfer-Asian Research, 7 pages, 2013.

[28] S. J. Liao, On the proposed homotopy analysis technique for nonlinear problems and its applications [Ph.D. thesis], Shanghai Jio Tong University, 1992. 


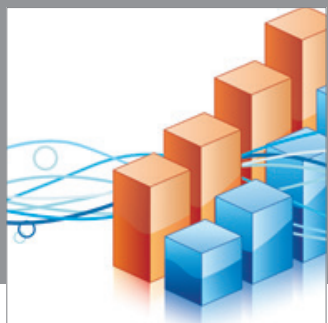

Advances in

Operations Research

mansans

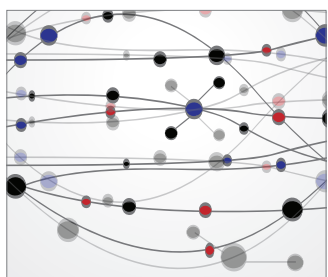

The Scientific World Journal
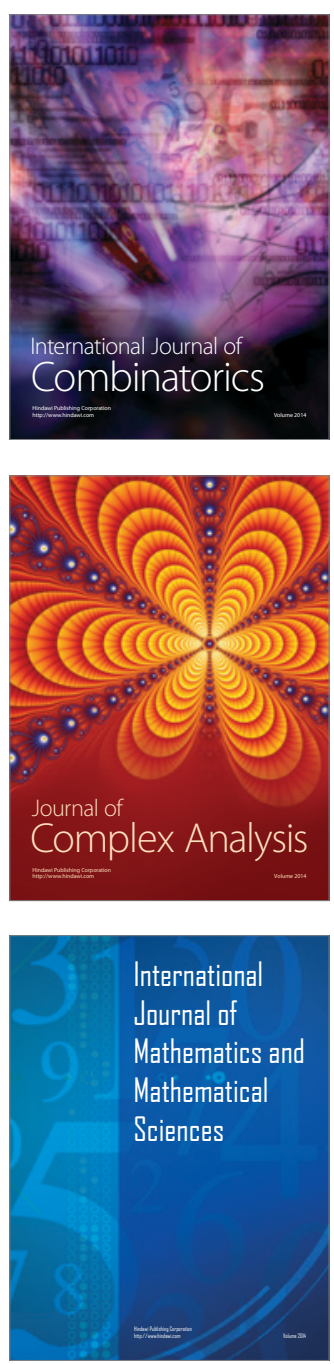
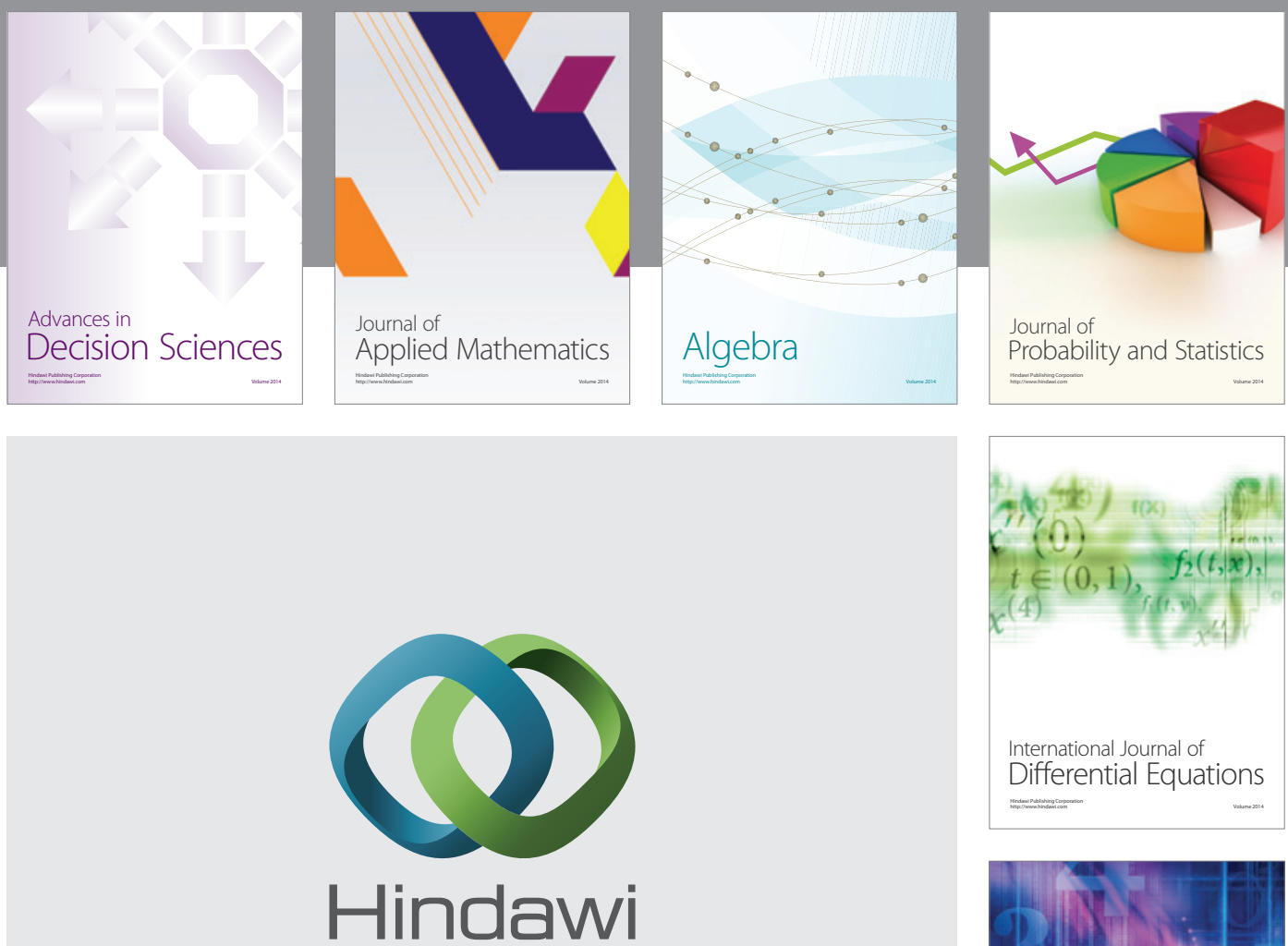

Submit your manuscripts at http://www.hindawi.com
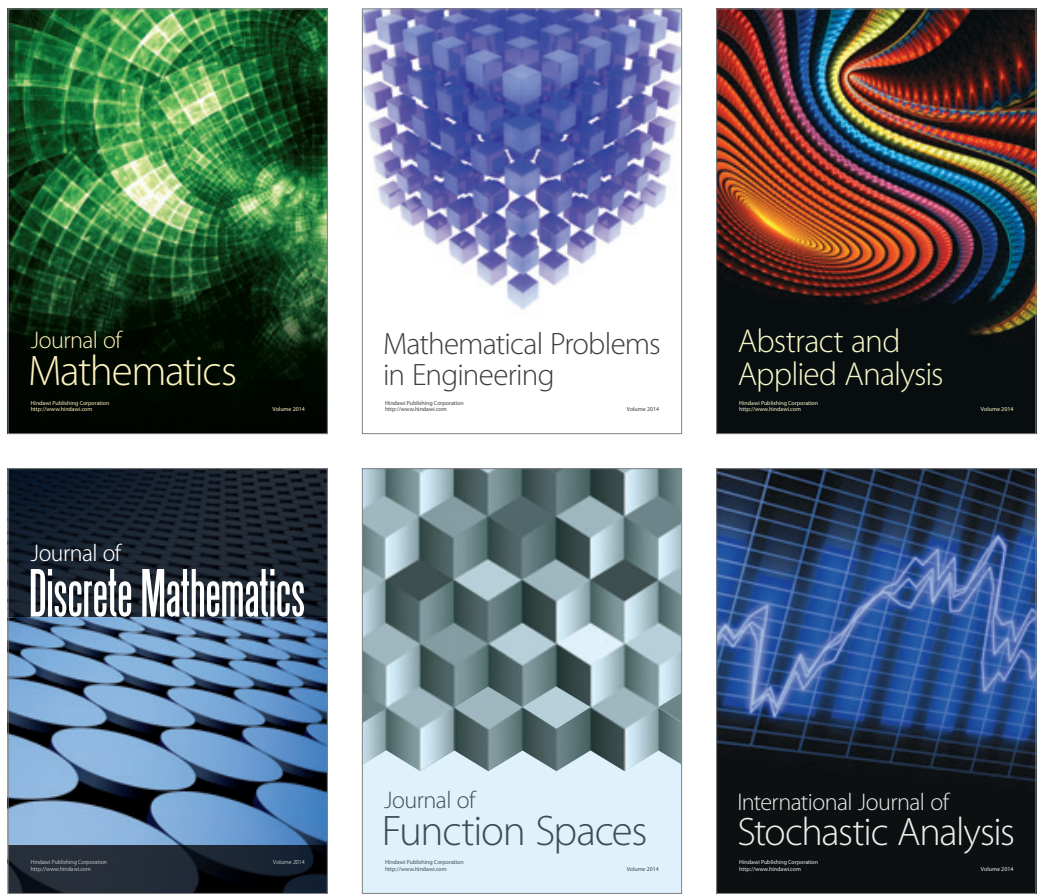

Journal of

Function Spaces

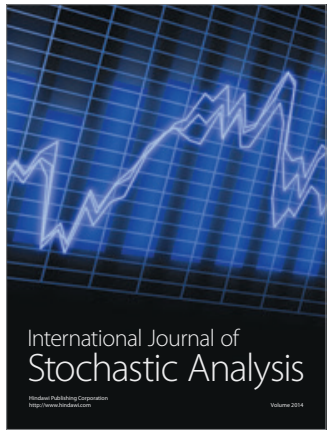

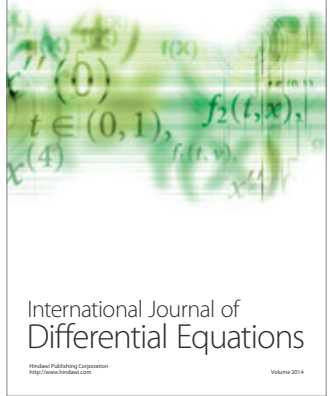
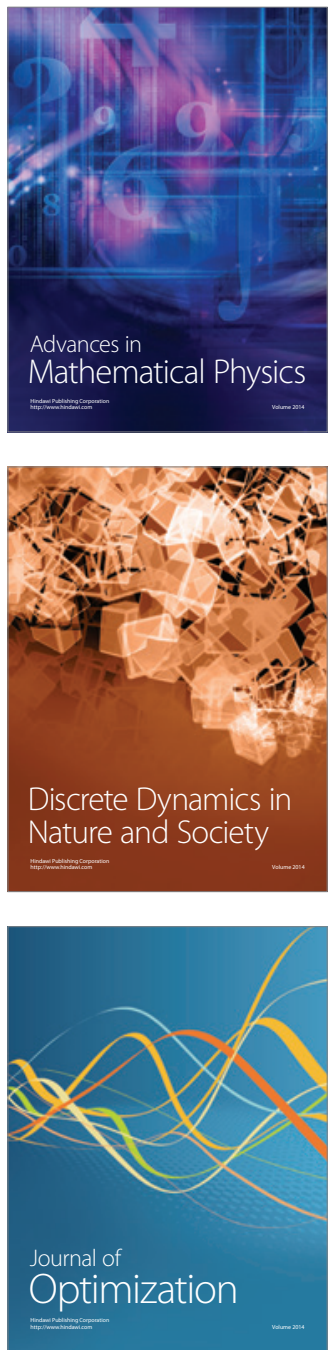